Discrete Comput Geom 29:61-75 (2003)

DOI: $10.1007 / \mathrm{s} 00454-002-2810-8$

\title{
A Space of Cyclohedra
}

\author{
Satyan L. Devadoss \\ Department of Mathematics, Ohio State University, \\ Columbus, OH 43210, USA \\ devadoss@math.ohio-state.edu
}

To the memory of Rodica Simion

\begin{abstract}
The real points of the Deligne-Knudsen-Mumford moduli space $\overline{\mathcal{M}}_{0}^{n}$ of marked points on the sphere have a natural tiling by associahedra. We extend this idea to construct an aspherical space tiled by cyclohedra. We explore the structure of this space, coming from blow-ups of hyperplane arrangements, as well as discuss possibilities of its role in knot theory and mathematical physics.
\end{abstract}

\section{Associahedra and Cyclohedra}

1.1. We present an overview of two important polytopes.

Definition 1.1.1. Let $\mathfrak{A}(n)$ be the poset of all dissections of a convex $(n+1)$-gon using non-intersecting diagonals, ordered such that $a \prec a^{\prime}$ if $a$ is obtained from $a^{\prime}$ by adding new diagonals. ${ }^{1}$ The associahedron $K_{n}$ is a convex polytope of $\operatorname{dim} n-2$ whose face poset is isomorphic to $\mathfrak{A}(n)$.

The construction of the polytope $K_{n}$ is given by Lee [14] and Haiman (unpublished). Stasheff originally defined the associahedron for use in homotopy theory in connection with associativity properties of $H$-spaces [18, Section 2]. There is a well-known bijection between dissections of a convex polygon and partial bracketings of letters. Figure 1 shows an example of this relationship.

\footnotetext{
${ }^{1}$ Mention of diagonals will henceforth mean non-intersecting ones.
} 

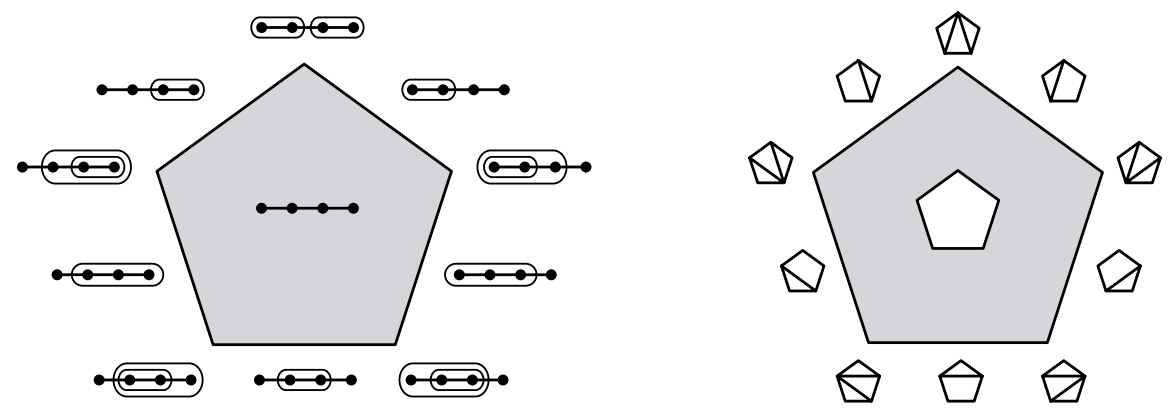

Fig. 1. Associahedron $K_{4}$ using brackets and polygons.

1.2. The cyclohedron $W_{n}$ originally manifested itself in the work of Bott and Taubes and later given its name by Stasheff. A construction of the polytope $W_{n}$ is given by Markl $[15$, Section 1] based on the following.

Definition 1.2.1. Let $\mathfrak{B}(n)$ be the poset of all partial bracketings of $n$ letters arranged on a circle, ordered such that $b \prec b^{\prime}$ if $b$ is obtained from $b^{\prime}$ by adding new pairs of brackets. The cyclohedron $W_{n}$ is a convex polytope of $\operatorname{dim} n-1$ whose face poset is isomorphic to $\mathfrak{B}(n)$.

It was the clever idea of Simion to come up with an alternate poset isomorphic to $\mathfrak{B}(n)$; in fact, she provides a construction of $W_{n}$ using this poset [17, Section 2]. It is formulated in terms of centrally symmetric $2 n$-gons, where a diagonal on such a polygon will either mean a pair of centrally symmetric diagonals or a diameter of the polygon.

Proposition 1.2.2. The poset of non-intersecting diagonals on a centrally symmetric $2 n$-gon, ordered such that $b \prec b^{\prime}$ if $b$ is obtained from $b^{\prime}$ by adding diagonals, is isomorphic to $\mathfrak{B}(n)$.
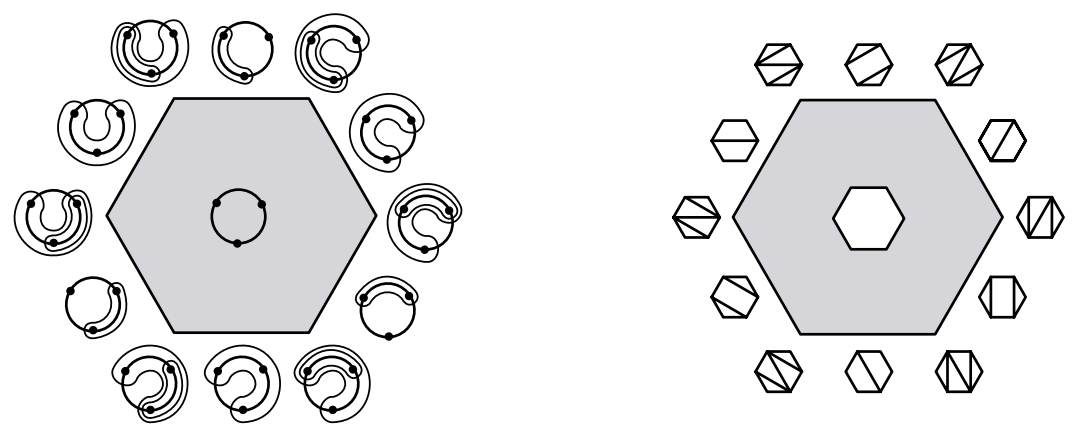

Fig. 2. Cyclohedron $W_{3}$ using brackets and polygons.

Figure 2 shows an example of the two descriptions of $W_{3}$. One difference between $K_{n}$ and $W_{n}$ is that for the codim 1 faces of the associahedron, we do not place brackets 
around all $n$ variables. In contrast, the cyclohedron allows this since one can distinguish the cyclic manner in which the $n$ variables are combined.

1.3. It is worthwhile exploring some properties of $W_{n}$ and its relationship with associahedra. It was noted by Stasheff that the boundary faces of $K_{n}$ can be identified with products of lower-dimensional associahedra [18, Section 2]. He makes a similar observation for $W_{n}$.

Proposition 1.3.1 [19, Section 4]. Faces of the cyclohedron are products of lowerdimensional cyclohedra and associahedra. In particular, all codim $k$ faces of $W_{n}$ have the form

$$
W_{n_{0}} \times K_{n_{1}} \times \cdots \times K_{n_{k}},
$$

over varying values of $1 \leq n_{0}<n$ and $1<n_{i} \leq n$ such that $\sum n_{i}=n+k$.

Example 1.3.2. Since $W_{5}$ is a four-dimensional polytope, we look at its possible codim 1 faces. They are given by the different ways of placing a diagonal in a 10gon. Figure 3 illustrates the four possible types: On the far left is the product $W_{4} \times K_{2}$; since $K_{2}$ is simply a point, the result is $W_{4}$ itself. The middle figures show $W_{3} \times K_{3}$ and $W_{2} \times K_{4}$, where on one hand the line segment is $K_{3}$ and on the other it is $W_{2}$. The last possible type is $W_{1} \times K_{5}$, which is simply $K_{5}$.
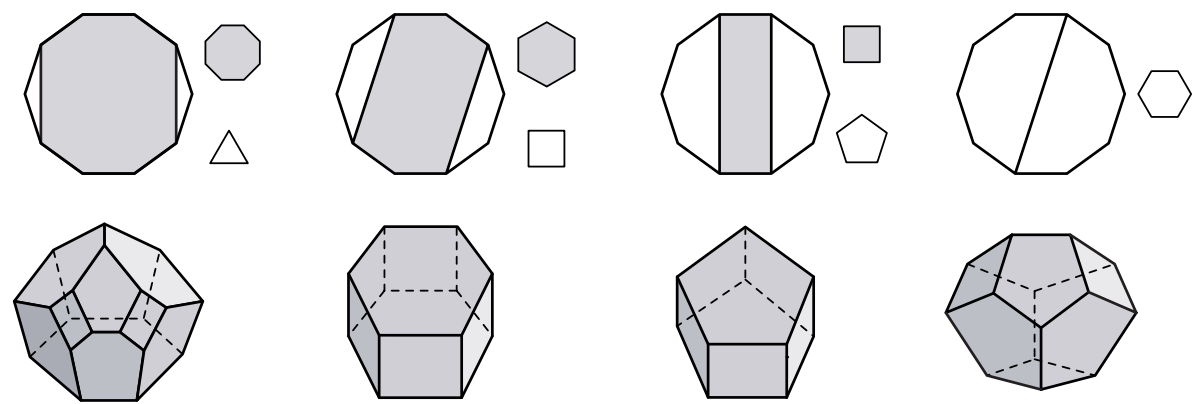

Fig. 3. Codim 1 faces of $W_{5}$.

Remark. A few observations follow:

1. For the associahedron, it is well known that the inclusions of lower-dimensional faces form the structure maps of an operad [18]. Stasheff and Markl show the inclusion maps above giving the cyclohedron a right module operad structure [15, Section 2].

2. The inclusion $K_{n} \hookrightarrow W_{n}$ coming from the proposition above shows the associahedron as a face of the cyclohedron. Tonks has constructed an explicit map 
$W_{n} \rightarrow K_{n+1}$ between polytopes of the same dimension [22], in the spirit of his map from the permutohedron $P_{n}$ to $K_{n+1}$ [21].

3. It is also noteworthy to point out a similar insight discovered by Ulyanov [23]. He shows $n$ copies of $K_{n+1}$ gluing together to form $W_{n}$. For example, the line segment $W_{2}$ is made up of two $K_{3}$ line segments with a pair of endpoints identified. Figure 4 shows the example of $W_{3}$ constructed using three copies of $K_{4}$, and, similarly, $W_{4}$ from four copies of $K_{5}$. Note that this gluing involves some non-trivial smoothing of adjacent faces after the gluing.
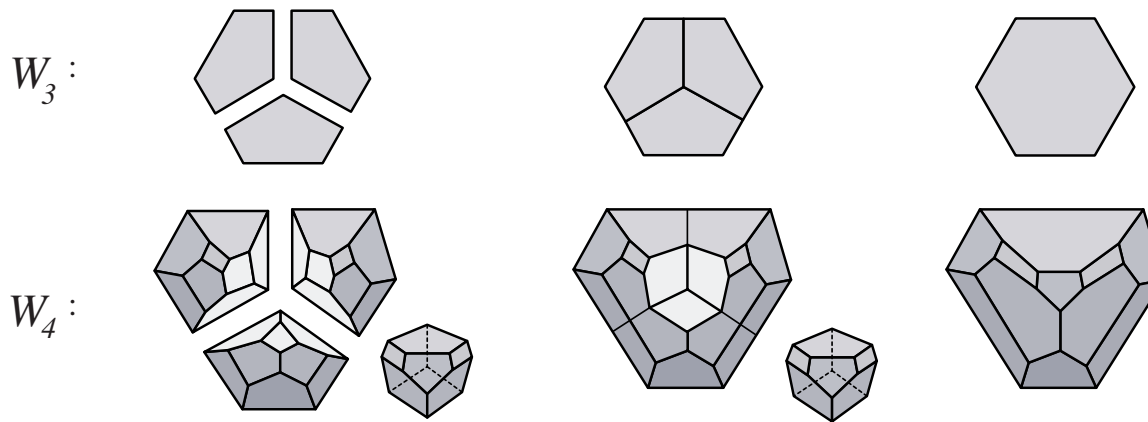

Fig. 4. Building $W_{3}$ and $W_{4}$ from copies of $K_{4}$ and $K_{5}$.

\section{Coxeter Groups and Blow-Ups}

2.1. The relationship between Coxeter groups and the polytopes $K_{n}$ and $W_{n}$ is introduced here. We begin by looking at certain hyperplane arrangements and refer the reader to [3] for any underlying terminology.

Definition 2.1.1. For a Euclidean space $V$, a linear hyperplane is a codim 1 subspace $H \subset V$ passing through the origin. A finite reflection group $W$ is a finite group of linear transformations of $V$ generated by reflections over a set of linear hyperplanes.

Let $V^{n} \subset \mathbb{R}^{n+1}$ be the hyperplane defined by $\Sigma x_{i}=0$. The collection of hyperplanes $\left\{x_{i}=x_{j} \mid i \neq j\right\}$ of $V^{n}$ forms the braid arrangement. The symmetric group $\mathbb{S}_{n+1}$ is the reflection group acting on $V^{n}$, where the transposition (ij) acts as orthogonal reflections across the hyperplane $x_{i}=x_{j}$ by permuting the coordinates. Let $\mathbb{S} V^{n}$ and $\mathbb{P} V^{n}$ respectively be the sphere and the projective space in $V^{n}$; that is, $\mathbb{P} V^{n}$ is isomorphic to $\mathbb{R} \mathbb{P}^{n-1}$. Note that the braid arrangement gives $\mathbb{P} V^{n}$ a $C W$-cellular decomposition into $\frac{1}{2}(n+1)$ ! open simplices. Figure 5(a) and (b) depicts the $n=3$ case.

The Coxeter (or Weyl) group of type $A_{n}$ is the symmetric group $\mathbb{S}_{n+1}$; moreover, it is the full symmetry group of the $n$-simplex, coming from representing the simplex as a convex linear combination of vectors. The Coxeter diagram

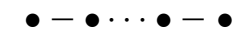




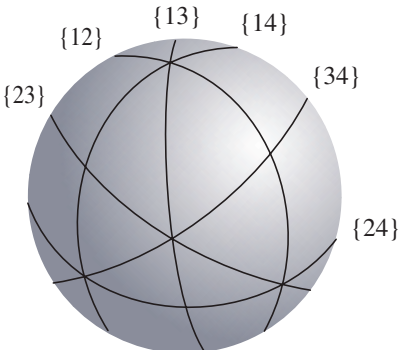

( a )

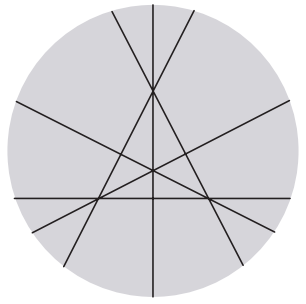

( b )

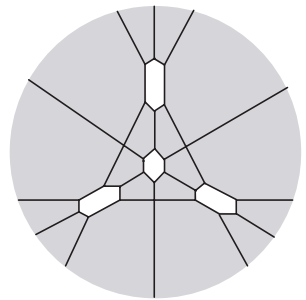

(c)

Fig. 5. $\mathbb{S} V^{3}, \mathbb{P} V^{3}$, and $\overline{\mathcal{M}}_{0}^{5}(\mathbb{R})$.

with $n$ nodes gives a presentation by $n$ transpositions $\left\{s_{i}\right\}$, with the relations $\left(s_{i} s_{i+1}\right)^{3}=$ 1 for adjacent generators and $\left(s_{i} s_{j}\right)^{2}=1$ otherwise. As discussed above, the braid arrangement dissects $\mathbb{P} V^{n}$ with the $(n-1)$-simplex as its fundamental chamber. The codim 1 faces of the simplex are indexed by the simple reflections, and hence by the nodes of the Coxeter diagram of $A_{n}$. The other faces are indexed by subdiagrams of the Coxeter diagram: a subdiagram gives a set of codim 1 faces, which intersect to give the indexed face.

Proposition 2.1.2. Truncating faces of the $(n-1)$-simplex which correspond to connected subdiagrams of the Coxeter diagram of $A_{n}$ in increasing order of dimension results in $K_{n+1}$. Furthermore, the associahedron is a simple polytope.

The proof follows from the associahedron construction given in Section 5 of [7]. Lee also obtains this using a sequence of stellar subdivisions on the polar dual to the associahedron [14, Section 3]. Note that the simplex is a simple polytope, and a truncation of a simple polytope remains simple. Figure 6 shows an example of the 3-simplex, with the initial truncation of vertices and then of edges, resulting in $K_{5}$.

2.2. We move from an associahedron to a space tiled by them. Let $M$ be a manifold and let $D \subset M$ be a union of codim 1 submanifolds which dissects $M$ into convex polytopes (chambers). The collection of hyperplanes $\left\{x_{i}=0 \mid i=1, \ldots, n\right\}$ of $\mathbb{R}^{n}$ generates the coordinate arrangement. A crossing (of $D$ ) in $M$ is normal if it is locally
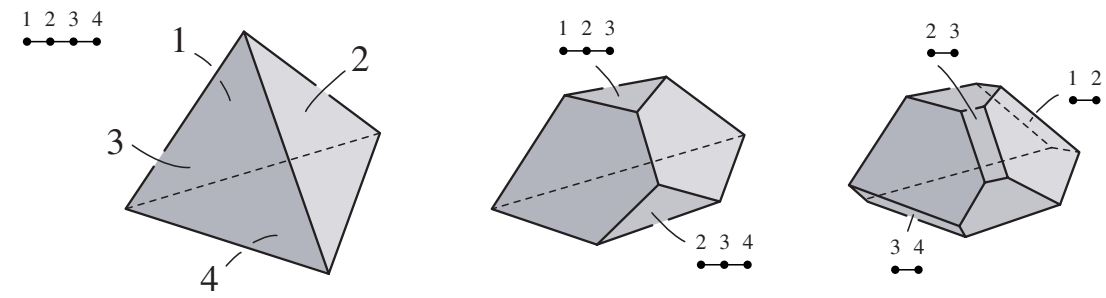

Fig. 6. Truncation of simplex to $K_{5}$. 
isomorphic to a coordinate arrangement. If every crossing is normal, then $M$ is rightangled. $^{2}$ A construction which transforms any crossing into a normal crossing involves the algebro-geometric concept of a blow-up.

Definition 2.2.1. For a linear subspace $X$ of a vector space $Y$, we blow-up $\mathbb{P} Y$ along $\mathbb{P} X$ by removing $\mathbb{P} X$, replacing it with the sphere bundle associated to the normal bundle of $\mathbb{P} X \subset \mathbb{P} Y$, and then projectifying the bundle.

A general collection of blow-ups is usually non-commutative in nature; in other words, the order in which spaces are blown up is important. For a given arrangement, De Concini and Procesi [6] establish the existence (and uniqueness) of a minimal building set, a collection of subspaces for which blow-ups commute for a given dimension, and for which the resulting space is right-angled. We use the definition found in Section 3 of [5].

Definition 2.2.2. A codim $k$ minimal element $\mathfrak{m}_{k}$ of the minimal building set of $\mathbb{P} V^{n}$ is the subspace indexed by a connected subdiagram of $A_{n}$ with $k$ nodes.

The Deligne-Knudsen-Mumford moduli space $\overline{\mathcal{M}}_{0}^{n}$ of marked points on the sphere is one of the central objects in mathematical physics. It is due to Kapranov that the connection between the braid arrangement and the real points $\overline{\mathcal{M}}_{0}^{n}(\mathbb{R})$ of this moduli space is made.

Theorem 2.2.3 [11, Section 4]. The iterated blow-up of $\mathbb{P} V^{n-2}$ along the cells $\left\{\mathfrak{m}_{k}\right\}$ in increasing order of dimension yields $\overline{\mathcal{M}}_{0}^{n}(\mathbb{R})$, with a tiling by $\frac{1}{2}(n-1)$ ! copies of $K_{n-1}$.

Example 2.2.4. Figure 5(b) and (c) shows $\mathbb{P} V^{3}$ before and after blowing up. The minimal elements of codim 2 turn out to be four points of triple intersection; blowing up along these components yield hexagons with antipodal identifications. Figure 5(b) has $\mathbb{R} \mathbb{P}^{2}$ tiled by 12 simplices; Fig. 5(c) is the connected sum of five real projective planes, tiled by $12 K_{4}$ pentagons.

2.3. We move from spherical geometry coming from linear hyperplanes to Euclidean geometry arising from affine hyperplanes. Let $\tilde{A}_{n}$ denote the affine root system of type $A_{n}$. The Coxeter diagram of $\tilde{A}_{n}$ is $n+1$ vertices arranged on a circle and its Coxeter group is the semi-direct product $\tilde{\mathbb{S}}_{n+1}=\mathbb{Z}^{n} \rtimes \mathbb{S}_{n+1}$, where the $n+1$ generators (transpositions) satisfy $\left(s_{i} s_{j}\right)^{3}=1$ when adjacent on the diagram and commute otherwise. Similar to above, let the nodes of the Coxeter diagram of $\tilde{A}_{n}$ correspond to the top dimensional faces of the $n$-simplex.

Proposition 2.3.1. Truncating faces of the $n$-simplex which correspond to connected subdiagrams of $\tilde{A}_{n}$ in increasing order of dimension results in $W_{n+1}$. Furthermore, the cyclohedron is a simple polytope.

\footnotetext{
${ }^{2}$ The dual cellulation of $M$ is a cubical cell complex: its cells are combinatorially equivalent to cubes.
} 


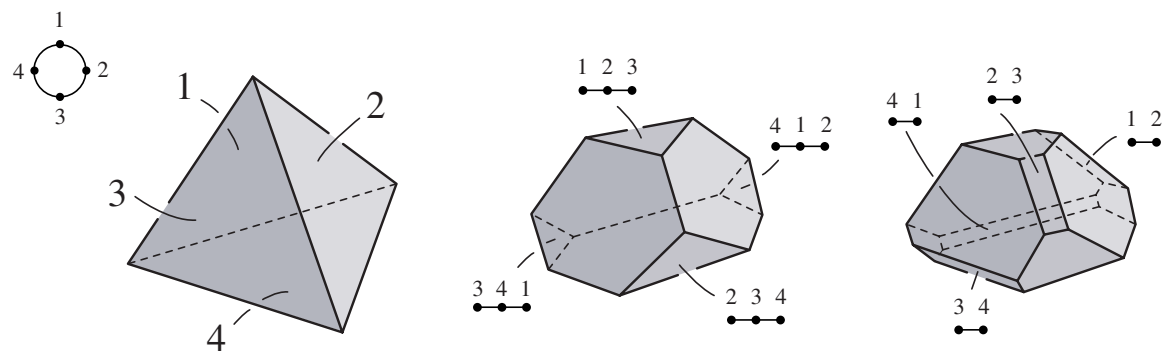

Fig. 7. Truncation of simplex to $W_{4}$.

The proof follows from the cyclohedron construction given in Appendix B of [19]. Figure 7 shows an example from $\tilde{A}_{3}$, with the truncation of vertices and edges, resulting in $W_{4}$.

Definition 2.3.2. An affine hyperplane of $V$ is a subset of the form $x+H$, where $x \in V$ and $H$ is a linear hyperplane of $V$. An affine reflection group $W$ is a locally finite group of affine isometries of $V$ generated by reflections over a set of affine hyperplanes.

We follow the construction given in Section 6 of [3]. Let $V^{n} \subset \mathbb{R}^{n}$ be the hyperplane defined by $\Sigma x_{i}=0$. The affine braid arrangement is the set $\left\{x_{i}=x_{j}+k \mid i \neq\right.$ $j, k \in \mathbb{Z}\}$ of affine hyperplanes in $V^{n}$, having $\tilde{\mathbb{S}}_{n}$ as its affine reflection group. This arrangement gives $V^{n}$ a CW-cellular decomposition into an infinite collection of open $(n-1)$-simplices. The left side of Fig. 8 gives the example when $n=3$ generated by the reflections $\{(12),(23),(13)\}$.

Previously, we looked to the projective sphere $\mathbb{P} V^{n}$ to give a compact space. In the affine case, the natural candidate is the quotient $\mathbb{L} V^{n}=V^{n} / \mathbb{Z}^{n-1}$, which is homeomorphic to the $(n-1)$-torus. Note that each chamber of $V^{n}$ corresponds to an infinite, cyclically repeating chain of inequalities

$\cdots<x_{i_{n}}+k_{n}-1<x_{i_{1}}+k_{1}<x_{i_{2}}+k_{2}<\cdots<x_{i_{n}}+k_{n}<x_{i_{1}}+k_{1}+1<x_{i_{2}}+k_{2}+1<\cdots$,

where $k_{i} \in \mathbb{Z}$. Quotienting by $\mathbb{Z}^{n-1}$ amounts to only remembering the cyclic ordering $i_{1} i_{2} \cdots i_{n}$. Therefore, there are $(n-1)$ ! chambers in $\mathbb{L} V^{n}$, each with a particular (cyclic)
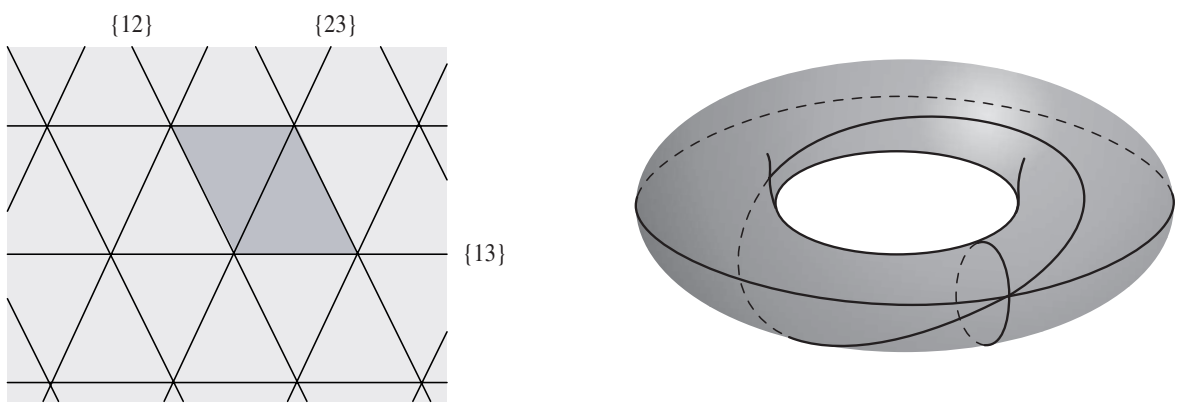

Fig. 8. Tiling of $\mathbb{R}^{2}$ and $\mathbb{L} V^{3}$. 
ordering. The right side of Fig. 8 shows the resulting $\mathbb{L} V^{3}$ tiled by two simplices. The affine braid arrangement also has the notion of a minimal building set. Recall that each face of an $(n-1)$-simplex tiling $\mathbb{L} V^{n}$ is indexed by a subdiagram of $\tilde{A}_{n-1}$.

Definition 2.3.3. A codim $k$ minimal element $\mathfrak{m}_{k}$ of the minimal building set of $\mathbb{L} V^{n}$ is the subspace indexed by a connected subdiagram of $\tilde{A}_{n-1}$ with $k$ nodes.

Remark. There is an alternate, combinatorial formulation for $\mathfrak{m}_{k}$. A connected, length $k$ subdiagram of $\tilde{A}_{n-1}$ is the Coxeter diagram $A_{k}$ having $\mathbb{S}_{k+1}$ as its Coxeter group. It follows that exactly $\left(\begin{array}{c}k+1 \\ 2\end{array}\right)$ hyperplanes intersect at $\mathfrak{m}_{k}$, with the transpositions of $\mathbb{S}_{k+1}$ generating reflections across these hyperplanes.

Definition 2.3.4. Let $\overline{\mathcal{Z}}^{n}$ be the iterated blow-up of $\mathbb{L} V^{n}$ along the cells $\left\{\mathfrak{m}_{k}\right\}$ in increasing order of dimension.

Corollary 2.3.5. $\quad \overline{\mathcal{Z}}^{n}$ is a manifold of dimension $n-1$ and is without boundary. Furthermore, $\overline{\mathcal{Z}}^{n}$ is tiled by $(n-1)$ ! copies of $W_{n}$ and is right-angled.

Proof. These properties are immediate from the construction of $\overline{\mathcal{Z}}^{n}$ above and Proposition 2.3.1. An alternate proof of $\overline{\mathcal{Z}}^{n}$ being right-angled is given below.

Example 2.3.6. The left side of Fig. 9 shows the result after blowing up the minimal vertex of $\mathbb{L} V^{3}$. We see a tiling by two $W_{3}$ hexagons, illustrated in detail on the right of the figure. The resulting $\overline{\mathcal{Z}}^{3}$ manifold is homeomorphic to $\mathbb{R} \mathbb{P}^{2} \# \mathbb{R} \mathbb{P}^{2} \# \mathbb{R} \mathbb{P}^{2}$.
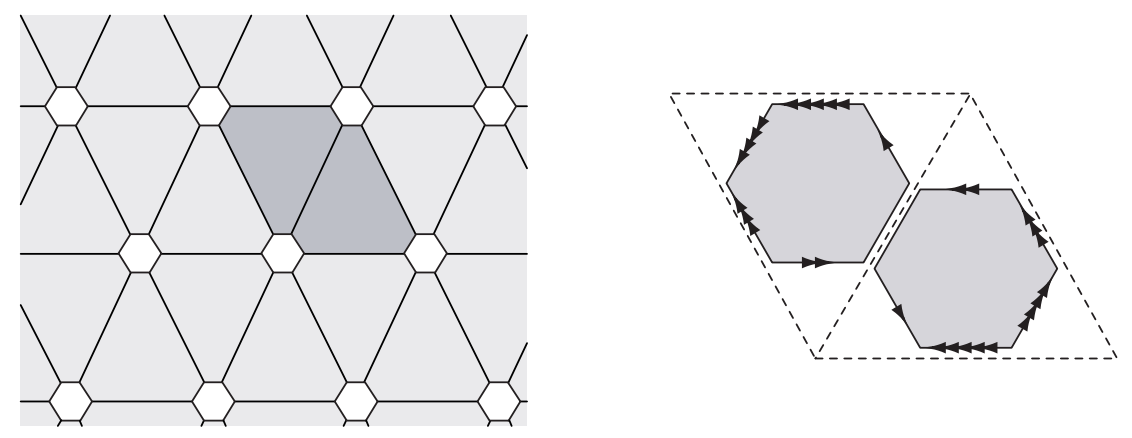

Fig. 9. Hexagons tiling $\overline{\mathcal{Z}}^{3}$.

\section{Tilings and Compactifications}

3.1. It was the combinatorial construction of $\overline{\mathcal{M}}_{0}^{n}(\mathbb{R})$ in Section 3 of [7] which prompted Simion to wonder about a space of cyclohedra. Although we use her definition of the cyclohedron, our results are independent of her work. 


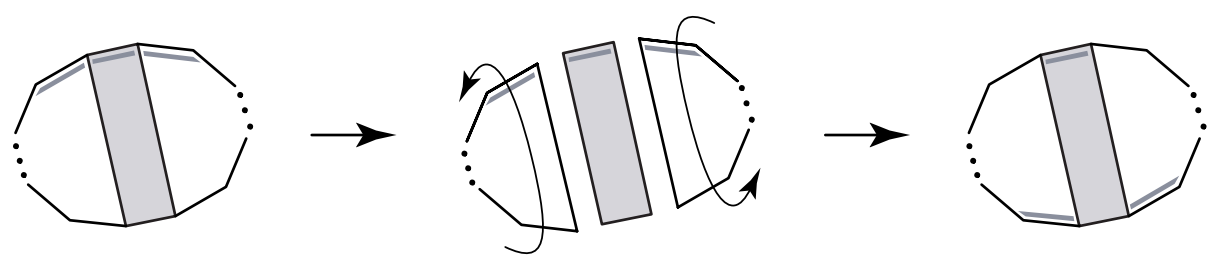

Fig. 10. Twist along a diagonal.

Definition 3.1.1. The twist of a $2 n$-gon $G$ along a (pair of centrally symmetric) diagonal $d$, denoted by $\nabla_{d}(G)$, is the polygon obtained by separating $G$ along $d$, twisting (reflecting) both pieces symmetrically, and gluing them back (Fig. 10).

Label the edges of a $2 n$-gon with $\{1,2, \ldots, n\}$ such that antipodal edges have the same label. Identify two such labeled polygons by a rotation action, yielding $(n-1)$ ! distinct polygons.

Theorem 3.1.2. Two centrally symmetric labeled $2 n$-gons $G_{1}, G_{2}$ with $k$ sets of diagonals (representing codim $k$ faces of cyclohedra) are identified in $\overline{\mathcal{Z}}^{n}$ if there exist diagonals $d_{1}, \ldots, d_{r}$ of $G_{1}$ such that

$$
\left(\nabla_{d_{1}} \cdots \nabla_{d_{r}}\right)\left(G_{1}\right)=G_{2}
$$

Proof. Recall that there are $(n-1)$ ! chambers in $\mathbb{L} V^{n}$, each with a particular cyclic ordering $i_{1} i_{2} \cdots i_{n}$. We encapsulate this information by assigning to each chamber a centrally symmetric $2 n$-gon, with sides labeled by the ordering $i_{1} i_{2} \cdots i_{n}$. The iterated blow-up of $\mathbb{L} V^{n}$ gives a tiling by cyclohedra, where the faces of $W_{n}$ correspond to labeled $2 n$-gons with diagonals. It remains to be checked that the gluing of these cyclohedra from minimal blow-ups is the same as using the twist operation. Without loss of generality, look at the fundamental chamber of $\mathbb{L} V^{n}$ defined by the ordering $12 \cdots n$. Each node of the Coxeter diagram of $\tilde{A}_{n-1}$ indexes a codim 1 face of this simplex, which belongs to a hyperplane of the form $x_{j}=x_{j+1}$. Let $\mathfrak{m}_{k}$ be a minimal cell in the boundary of this chamber. Since the subdiagram associated to $\mathfrak{m}_{k}$ is connected, this cell occurs at such intersections $x_{i}=x_{i+1}=\cdots=x_{i+k}$ of hyperplanes. ${ }^{3}$ Indeed, blowing up the cell seeks exactly to resolve the order in which collisions occur at such intersections. Crossing from the fundamental chamber through $\mathfrak{m}_{k}$ into its antipodal one in the arrangement corresponds to reflecting the element $i(i+1) \cdots(i+k)$ in the (cyclic) ordering $12 \cdots n$. Blowing up the minimal cell $\mathfrak{m}_{k}$ identifies cyclohedral faces across the antipodal chambers. Indeed, our notation of labeled polygons with twisting along diagonals mimics gluing antipodal faces after blow-ups.

Example 3.1.3. Figure 11 demonstrates the construction of $\overline{\mathcal{Z}}^{2}$ from one copy of $W_{2}$ to form $S^{1}$. The right side of Fig. 9 shows the gluing of two $W_{3}$ hexagons resulting in $\overline{\mathcal{Z}}^{3}$.

${ }^{3}$ Therefore, each $\mathfrak{m}_{k}$ corresponds to choosing $k+1$ elements from a set $\left\{x_{1}, \ldots, x_{n}\right\}$ of order $n$. 

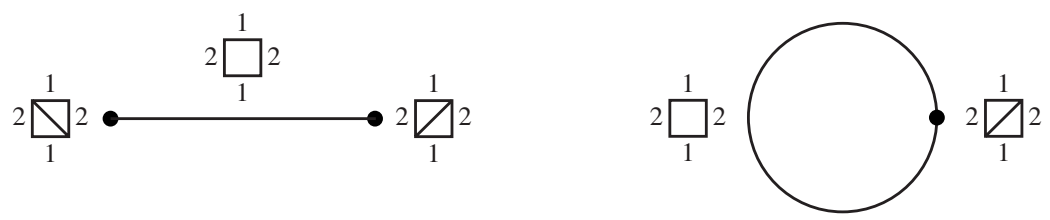

Fig. 11. $W_{2}$ and $\overline{\mathcal{Z}}^{2}$.

Corollary 3.1.4. $\quad \overline{\mathcal{Z}}^{n}$ is right-angled.

Proof. Fix a codim $k$ face of some cyclohedron in $\overline{\mathcal{Z}}^{n}$, represented by a labeled centrally symmetric $2 n$-gon with $k$ diagonals. Each twist along a diagonal moves to another cyclohedron adjacent to this face. There are $2^{k}$ such possible combinations of twists, giving a normal crossing at each face.

Remark. Faces of the associahedron are decomposed into the categories of dimension, type, and class, where they are used to understand the structure of $\overline{\mathcal{M}}_{0}^{n}(\mathbb{R})$ better (see Section 2 of [8] for definitions and details). For example, the enumeration of faces with respect to dimension leads to the Euler characteristic of $\overline{\mathcal{M}}_{0}^{n}(\mathbb{R})$. Using methods and results from [8], the initiated reader could easily carry out similar combinatorial calculations for the cyclohedron to describe properties of $\overline{\mathcal{Z}}^{n}$ further.

3.2. Our motivation in further analyzing $\overline{\mathcal{Z}}^{n}$ comes from some observations in Fig. 9 . The minimal vertex after blow-up becomes a hexagon with antipodal identification. This is essentially a triangle, which can be seen as $\overline{\mathcal{M}}_{0}^{4}(\mathbb{R})$. Note also that the three lines on $\mathbb{L} V^{3}$ become line segments with endpoint identifications in $\overline{\mathcal{Z}}^{3}$, which is seen from Fig. 11 as three copies of $\overline{\mathcal{Z}}^{2}$. In general, we observe the following:

Theorem 3.2.1. There are $\left(\begin{array}{c}n \\ k+1\end{array}\right)$ copies of $\overline{\mathcal{M}}_{0}^{k+2}(\mathbb{R}) \times \overline{\mathcal{Z}}^{n-k}$ in $\overline{\mathcal{Z}}^{n}$.

Proof. We show that the blow-up of a minimal element $\mathfrak{m}_{k}$ of $\mathbb{L} V^{n}$ results in $\overline{\mathcal{M}}_{0}^{k+2}(\mathbb{R}) \times$ $\overline{\mathcal{Z}}^{n-k}$. As discussed above, each $\mathfrak{m}_{k}$ corresponds to choosing $k+1$ elements from a set $\{1, \ldots, n\}$. Choose an arbitrary minimal cell and assign it such a choice, say $\left\{p_{1}, \ldots, p_{k+1}\right\}$. We view this as a centrally symmetric $2 n$-gon having a diagonal partitioning it into a pair of symmetrically labeled sides $\left\{p_{1}, \ldots, p_{k+1}\right\}$, with the sides of the central polygon using the remaining labels. Note the correspondence of this dissected $2 n$-gon to the product $K_{k+1} \times W_{n-k}$.

As this minimal cell is blown-up, a system records the different ways points approach it; this corresponds to keeping track of the order of labels placed on the polygon. Since twisting is allowed along diagonals, there are $\frac{1}{2}(k+1)$ ! ways to arrange labels on the non-central polygons, each corresponding to a $K_{k+1}$. Indeed, this is exactly how one gets $\overline{\mathcal{M}}_{0}^{k+2}(\mathbb{R})$, with the associahedra glued as defined above. Therefore, a fixed labeling of the central polygon gives $\overline{\mathcal{M}}_{0}^{k+2}(\mathbb{R}) \times W_{n-k}$, while allowing all possible labelings yields the result. 

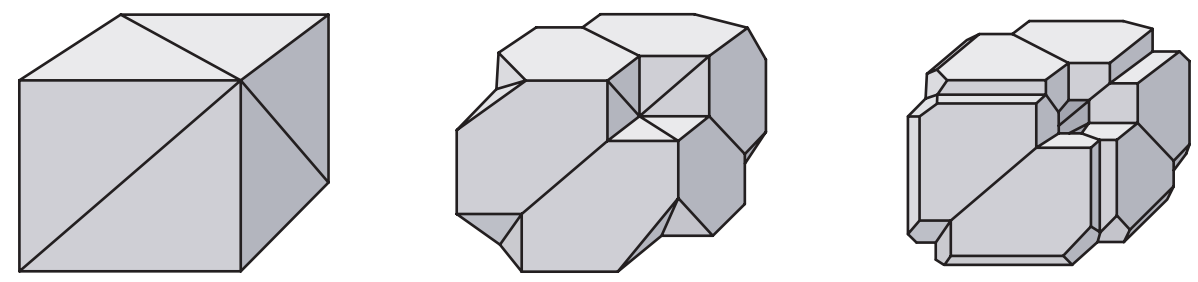

Fig. 12. Blow-ups leading to six $W_{4}$ polytopes tiling $\overline{\mathcal{Z}}^{4}$.

Example 3.2.2. We move to the $n=4$ case to illustrate the theorem further. The leftmost drawing of Fig. 12 shows the torus $\mathbb{L} V^{4}$ as the fundamental cube tiling $\mathbb{R}^{3}$ with hyperplane cuts. Blowing up the minimal vertex (codim 3$)$ and then the lines (codim 2$)$ yield the middle and right-hand pictures. The space $\overline{\mathcal{Z}}^{4}$ is shown to be tiled by six copies of $W_{4}$. Notice the vertices of the cube (identified to form a single vertex of the 3torus) become $\overline{\mathcal{M}}_{0}^{5}(\mathbb{R})$ after blow-ups, tiled by 12 pentagons. The minimal lines become triangular tori, that is, $\overline{\mathcal{M}}_{0}^{4}(\mathbb{R}) \times \overline{\mathcal{Z}}^{2}$. A face of the cube can be seen as a 2-torus with blow-ups, resulting in $\overline{\mathcal{Z}}^{3}$ tiled by two $W_{3}$ hexagons.

3.3. The original definition of $W_{n}$ describes it in terms of a compactified configuration space of points on the circle [2, Section 3]. We extend this to $\overline{\mathcal{Z}}^{n}$. A configuration space of $n$ labeled points on an $m$-manifold $X$ is defined as

$$
\mathrm{F}(X, n)=X^{n}-\Delta, \quad \text { where } \quad \Delta=\left\{\left(x_{1}, \ldots, x_{n}\right) \in X^{n} \mid \exists i, j, x_{i}=x_{j}\right\} .
$$

Compactifying $\mathrm{F}(X, n)$ enables the points to collide and a system is introduced to record the directions points arrive at the collision. In the work of Fulton and MacPherson [9], this method is brought to rigor in the algebro-geometric context. ${ }^{4}$ As points collide, they land on a screen, viewed as $\mathbb{R} \mathbb{P}^{m}$, which is identified with the point of collision. ${ }^{5}$ Now these points on the screen are themselves allowed to move and collide, landing on higher level screens. However, they are free up to an action of $\mathbb{P G l}_{m+1}(\mathbb{R})$, the affine automorphism on each screen. Kontsevich describes the process in terms of a magnifying glass: on any given level, only a configuration of points is noticeable; but one can zoom-in on a particular point and peer into its screen, seeing the space of collided points. As mentioned above, for an arrangement of hyperplanes, De Concini and Procesi develop a method to compactify their complements by iterated blow-ups using a minimal building set. In the case of the arrangement $X^{n}-\mathrm{F}(X, n)$, their procedure yields the Fulton-MacPherson compactification of $\mathrm{F}(X, n)[6]$.

Theorem 3.3.1. $\quad \overline{\mathcal{Z}}^{n}$ is a real Fulton-MacPherson compactification of $\mathrm{F}\left(S^{1}, n\right)$ quotiented by the action of $S^{1}$.

Proof. The affine braid arrangement $\left\{x_{i}=x_{j}+k \mid i \neq j, k \in \mathbb{Z}\right\}$ decomposes $V^{n} \subset \mathbb{R}^{n}$ into $(n-1)$-simplices. Let $H_{i}$ denote the hyperplane $x_{1}=x_{i}$ and let $V_{i}$ be

\footnotetext{
${ }^{4}$ Fulton-MacPherson is over the complexes, but for our purposes the reals are more relevant.

${ }^{5}$ These projective spheres have been dubbed bubbles, and the compactification process as bubbling.
} 
the one-dimensional subspace of $V^{n}$ defined by $H_{2} \cap \cdots \cap \hat{H}_{i} \cap \cdots \cap H_{n}$, where the symbol $\hat{H}_{i}$ means the hyperplane $H_{i}$ is to be deleted from the intersection. If $v_{i}$ is a unit vector in $V_{i}$ emanating from the origin, then $\left\{v_{i} \mid i=2, \ldots, n\right\}$ forms a set of $\mathbb{Z}$-basis vectors for a lattice. Without loss of generality, obtain the $(n-1)$-torus $\mathbb{L} V^{n}$ from $V^{n}$ by modding out by this lattice.

On the other hand, since $S^{1}$ acts freely on $\mathrm{F}\left(S^{1}, n\right)$, its quotient simply fixes one of the $n$ points, say $x_{1}=1 \in S^{1}$, resulting in $\left(S^{1}\right)^{n-1}-\Delta^{\prime}$, where

$$
\Delta^{\prime}=\left\{\left(x_{2}, \ldots, x_{n}\right) \in\left(S^{1}\right)^{n-1} \mid \exists i, j, x_{i}=x_{j} \text { or } x_{i}=1\right\} .
$$

It follows that the hyperplane markings on $\mathbb{L} V^{n}$ coincide with the arrangement $\Delta^{\prime}$ on the $(n-1)$-torus. Therefore, from the discussion above, the Fulton-MacPherson compactification of $\mathrm{F}\left(S^{1}, n\right) / S^{1}$ coincides with the blow-ups of the minimal building set of $\mathbb{L} V^{n}$.

Remark. To see this for $n=3$, note that $\mathrm{F}\left(S^{1}, 3\right) / S^{1}$, viewed as the front face of the cube in Fig. 12, is identical to $\mathbb{L} V^{3}$, the shaded region of Fig. 8.

The appearance of cyclohedra in the Fulton-MacPherson compactification above can intuitively be seen as follows: The compactification distinguishes the base space $S^{1}$ from the bubbles $\mathbb{R} \mathbb{P}^{1}$ coming from collisions. We keep track of this information by shading in the circle corresponding to the base space, shown in Fig. 13(a). There is a bijection between these bubble-trees and partial bracketings of $n$ points on $S^{1}$, which can be expressed in terms of $2 n$-gons with centrally symmetric diagonals (Fig. 13(b) and (c)), demonstrating the appearance of $W_{n}$. Note how the collision of points is represented as drawing a diagonal and its symmetric counterpart. There is a $\mathbb{Z}_{2}$ reflection in $\mathbb{P G l}_{2}(\mathbb{R})$ acting on each $\mathbb{R P}^{1}$ bubble (coming from the affine automorphisms on each screen) which corresponds to twisting along diagonals of the $2 n$-gons.

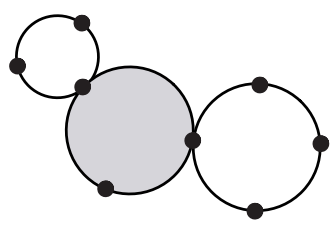

( a )

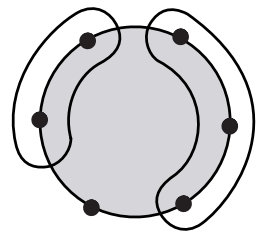

( b )

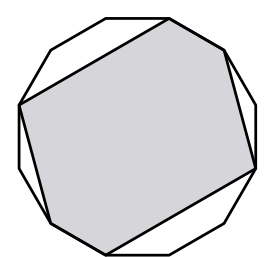

(c)

Fig. 13. Bubbles and centrally symmetric polygons.

\section{Some Observations}

4.1. We have shown the cyclohedron to be the $\tilde{A}_{n}$ affine analogue of the associahedron. However, Simion thought of it as a type $B_{n}$ associahedron. The reasoning behind this is explained by looking at the combinatorial question that motivated her. We thank Reiner for helpful insights into his work [16]. 
Definition 4.1.1. A $k$-partition separates $\{1, \ldots, n\}$ into disjoint sets $B_{1}, \ldots, B_{k}$. A partition is non-crossing if for elements $x_{1}, x_{2} \in B_{i}$ and $y_{1}, y_{2} \in B_{j}$ such that $x_{1}<$ $y_{1}<x_{2}<y_{2}$, then $i=j$. Let $N C(n, k)$ be the number of non-crossing $k$-partitions of $\{1, \ldots, n\}$.

The $f$-vector $\left\{f_{i}\right\}$ of a polytope $P$ is a combinatorial invariant, where $f_{k}(P)$ equals the number of $k$-dimensional faces. Another method of encapsulating this information is by the $h$-vector $\left\{h_{i}\right\}$ (see [24] for details). Denoting $K_{n}^{*}$ as the combinatorial dual of the associahedron, the following remarkable equivalence exists:

$$
N C(n, n-k)=h_{k}\left(K_{n+1}^{*}\right) .
$$

The poset of partitions of $\{1, \ldots, n\}$ is isomorphic to the lattice of intersection subspaces for the type $A_{n}$ braid arrangement. For example, a partition $\{1,2,4\}\{3,5\}$ corresponds to the intersection subspace of $x_{1}=x_{2}=x_{4}$ and $x_{3}=x_{5}$. Similarly, by replacing the set with $\{1, \ldots, n, \overline{1}, \ldots, \bar{n}\}$, we can consider the hyperoctahedral hyperplane arrangement for type $B_{n}$. The barring is an involution denoting the sign change coming from the hyperplanes $x_{i}= \pm x_{j}$.

Definition 4.1.2. A $k_{B}$-partition separates $\{1, \ldots, n, \overline{1}, \ldots, \bar{n}\}$ into disjoint sets $B_{0}$, $B_{1}, \ldots, B_{k}, \bar{B}_{1}, \ldots, \bar{B}_{k}$, where $\bar{B}_{i}=\left\{\bar{x} \mid x \in B_{i}\right\}$ and $B_{i}=\bar{B}_{i}$ only when $i=0$.

Let $N C_{B}(n, k)$ be the number of non-crossing $k_{B}$-partitions of $\{1, \ldots, n, \overline{1}, \ldots, \bar{n}\}$. Simion was able to show that the cyclohedron satisfies the equality

$$
N C_{B}(n, n-k)=h_{k}\left(W_{n+1}^{*}\right) .
$$

Remark. Combinatorially, Simion [17, Section 4] was able to construct a space tiled by eight copies of $W_{3}$ hexagons using the twist operation defined in [7]. We now see that she had arrived at the fourfold cover of $\overline{\mathcal{Z}}^{3}$ (in Fig. 8, choose a region four times that of the shaded one). By labeling the antipodal sides of the polygons with $i$ and $\bar{i}$, a $2^{n}$-fold cover of the moduli space $\overline{\mathcal{Z}^{n}}$ is created.

Remark. Burgiel and Reiner discuss two more type $B_{n}$ analogues of the associahedron, both being different from the cyclohedron [4].

4.2. The cyclohedron made its debut in knot theory implicitly through the work of Kontsevich [13] and explicitly by Bott and Taubes [2]. The embedding $S^{1} \rightarrow \mathbb{R}^{3}$ defining a knot induces a map onto their compactified $n$ point configuration spaces, thereby introducing $W_{n}$. Roughly, one then "carefully" integrates certain pullbacks of volume forms on $S^{2}$ using the inclusion $S^{1} \rightarrow \mathbb{R}^{3} \rightarrow S^{2}$; for a good topological understanding, see the work of Thurston [20].

The cyclohedron also appears in the work of Bar-Natan in a different setting [1, Section 4]. He gives a combinatorial approach of sketching some relations that arise in computing certain Kontsevich-KZ invariants coming from braids and chord diagrams 
(note that chord diagrams come from $2 n$ marked points on $S^{1}$ with pairwise identification). He introduces a multitude of relations and their corresponding polytopal structures, including $W_{n}$ and Kapranov's permutoassociahedron $K P_{n}$.

4.3. If a manifold is right-angled, its dual cellulation is a cubical cell complex. Gromov beautifully relates a combinatorial condition of such spaces with non-positive curvature [10]. A simplical complex $M$ is a flag complex if any finite collection of pairwise connected vertices spans a simplex in $M$.

Lemma 4.3.1 [Gromov]. The natural piecewise Euclidean metric on a cubical complex is non-positively curved if and only if the link of every vertex is a flag complex.

Gromov has also shown that the universal cover of a non-positively curved space is contractible. In other words, the homotopy properties of these spaces are completely encapsulated in their fundamental groups. Davis et al. apply Gromov's ideas to minimal blow-ups of certain hyperplane arrangements, proving the following:

Theorem 4.3.2 [5, Section 5]. The moduli spaces $\overline{\mathcal{M}}_{0}^{n}(\mathbb{R})$ and $\overline{\mathcal{Z}}^{n}$ are aspherical.

Recall that the Artin group of $A_{n}$ is the classic braid group on $n+1$ strings between points on an interval (Fig. 14(a)) or on a circle (Fig. 14(b)); simply cut the circles open and lay the strings flat to obtain an isomorphism. The affine analog of classical braids is the Artin group of $\tilde{A}_{n}$; this can be presented as braids with $n+1$ strands restricted to a cylindrical shell. The latter diagrams in Fig. 14 show two distinct elements of the $\tilde{A}_{2}$ braid group. There is a close similarity between the fundamental group of $\overline{\mathcal{M}}_{0}^{n}(\mathbb{R})$ and the braid group, described from a combinatorial viewpoint in Section 6 of [7]. It should be possible to extend this to the affine case.

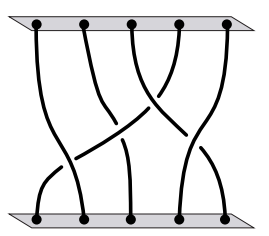

(a)

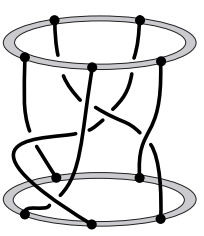

(b)

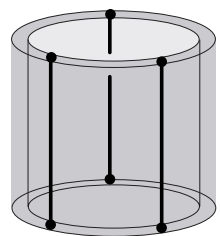

(c)

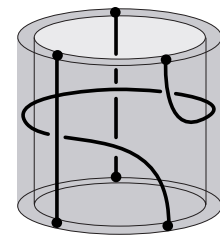

(d)

Fig. 14. Four braids, two coming from $A_{4}$ and two from $\tilde{A}_{2}$.

4.4. It seems quite natural to generalize what we have done. The motivation for the real moduli space of points $\overline{\mathcal{M}}_{0}^{n}(\mathbb{R})$ comes from its complex counterpart $\overline{\mathcal{M}}_{0}^{n}$. The importance of $\overline{\mathcal{M}}_{0}^{n}$ can be seen, for example, as being a fundamental building block in the theory of Gromov-Witten invariants, among numerous other appearances in literature. A reasonable candidate for the complex analogue for $\overline{\mathcal{Z}}^{n}$ could be the Fulton-MacPherson compactification of $\mathrm{F}\left(S^{2}, n\right) / S O(3)$. Again, we would like to know the role this space would play in mathematical physics and algebraic geometry. Similarly, we can generalize this construction to other Coxeter groups. Much has been done in terms of the minimal blow- 
ups in [5]. However, there have been a wealth of new compactifications that have been studied recently and we discuss the interplay between these ideas in a forthcoming work.

\section{Acknowledgments}

I am grateful to Mike Davis for pointing out the affine braid arrangement, to Vic Reiner for numerous insightful conversations, and to the referees for their thorough critique. Thanks also go to Jack Morava and Jim Stasheff for their encouragement.

\section{References}

1. D. Bar-Natan, The fundamental theorem of Vassiliev invariants, in Geometry and Physics (J.E. Andersen, J. Dupont, H. Pedersen, and A. Swann, eds.), pp. 101-134. Lecture Notes in Pure and Applied Mathematics, Vol. 184, Marcel Dekker, New York, 1997.

2. R. Bott and C. Taubes, On the self-linking of knots. J. Math. Phys. 35 (1994), 5247-5287.

3. K. S. Brown, Buildings. Springer-Verlag, New York, 1989.

4. H. Burgiel and V. Reiner, Two signed associahedra. New York J. Math. 4 (1998), 83-95.

5. M. Davis, T. Januszkiewicz, and R. Scott, Nonpositive curvature of blowups. Selecta Math. 4 (1998), 491-547.

6. C. De Concini and C. Procesi, Wonderful models of subspace arrangements. Selecta Math. 1 (1995), 459-494.

7. S. L. Devadoss, Tessellations of moduli spaces and the mosaic operad, in Homotopy Invariant Algebraic Structures, pp. 91-114. Contemporary Mathematics, Vol. 239, AMS, Providence, RI, 1999.

8. S. L. Devadoss and R. C. Read, Cellular structures determined by polygons and trees. Ann. Combin. 5 (2001), 71-98.

9. W. Fulton and R. MacPherson, A compactification of configuration spaces. Ann. Math. 139 (1994), 183225.

10. M. Gromov, Hyperbolic groups, in Essays in Group Theory, pp. 75-263. M.S.R.I. Publications, Vol. 8, Springer-Verlag, New York, 1987.

11. M. M. Kapranov, The permutoassociahedron, MacLane's coherence theorem, and asymptotic zones for the KZ equation. J. Pure Appl. Algebra 85 (1993), 119-142.

12. F. Knudsen, The projectivity of the moduli space of stable curves, II. Math. Scand. 52 (1983), 161-199.

13. M. Kontsevich, Feynman diagrams and low-dimensional topology. Progr. Math. 120 (1994), 97-121.

14. C. Lee, The associahedron and triangulations of the $n$-gon. European J. Combin. 10 (1989), 551-560.

15. M. Markl, Simplex, associahedron, and cyclohedron, in Higher Homotopy Structures in Topology and Mathematical Physics, pp. 235-265. Contemporary Mathematics, Vol. 227, AMS, Providence, RI, 1999.

16. V. Reiner, Non-crossing partitions for classical reflection groups. Discrete Math. 177 (1997), 195-222.

17. R. Simion, A type-B associahedron. Adv. Appl. Math., in press.

18. J. D. Stasheff, Homotopy associativity of $H$-spaces. Trans. Amer. Math. Soc. 108 (1963), 275-292.

19. J. D. Stasheff, The pre-history of operads, in Operads: Proceedings of Renaissance Conferences, pp. 53-81. Contemporary Mathematics, Vol. 202, AMS, Providence, RI, 1997.

20. D. Thurston, Integral expressions for the Vassiliev knot invariants, preprint math.AG/9901110v2.

21. A. Tonks, Relating the associahedron and the permutohedron, in Operads: Proceedings of Renaissance Conferences, pp. 33-36. Contemporary Mathematics, Vol. 202, AMS, Providence, RI, 1997.

22. A. Tonks, personal communication, 2000.

23. A. P. Ulyanov, personal communication, 2000.

24. G. M. Ziegler, Lectures on Polytopes. Graduate Texts in Mathematics, Vol. 152, Springer-Verlag, New York, 1995

Received March 8, 2001, and in revised form August 31, 2001, March 20, 2002, and April 30, 2002.

Online publication November 21, 2002. 